EPJ manuscript No.

(will be inserted by the editor)

\title{
Pygmy dipole strength close to particle-separation energies - the case of the Mo isotopes
}

\author{
Gencho Rusev ${ }^{1}$, Eckart Grosse ${ }^{1,2}$, Martin Erhard ${ }^{1}$, Arnd Junghans ${ }^{1}$, Krasimir Kosev ${ }^{1}$, Klaus-Dieter Schilling ${ }^{1}$, \\ Ronald Schwengner ${ }^{1}$ and Andreas Wagner ${ }^{1}$ \\ 1 Forschungszentrum Rossendorf, Institut für Kern- und Hadronenphysik, \\ Postfach 510119, 01314 Dresden Germany \\ 2 Technische Universität Dresden, Institut für Kern- und Teilchenphysik, \\ 01062 Dresden, Germany \\ Received: date / Revised version: date
}

\begin{abstract}
The distribution of electromagnetic dipole strength in ${ }^{92,98,100}$ Mo has been investigated by photon scattering using bremsstrahlung from the new ELBE facility. The experimental data for well separated nuclear resonances indicate a transition from a regular to a chaotic behaviour above $4 \mathrm{MeV}$ of excitation energy. As the strength distributions follow a Porter-Thomas distribution much of the dipole strength is found in weak and in unresolved resonances appearing as fluctuating cross section. An analysis of this quasi-continuum - here applied to nuclear resonance fluorescence in a novel way - delivers dipole strength functions, which are combining smoothly to those obtained from $(\gamma, \mathrm{n})$-data. Enhancements at $6.5 \mathrm{MeV}$ and at $\sim 9 \mathrm{MeV}$ are linked to the pygmy dipole resonances postulated to occur in heavy nuclei.
\end{abstract}

PACS. 21.10.Pc $-25.20 .-\mathrm{x}-25.20 . \mathrm{Dc}-26.30 .+\mathrm{k}$

\section{Dipole strength in heavy nuclei}

The response of nuclei to dipole radiation is of special importance for the synthesis of the chemical elements in the cosmos: Particle thresholds may be crossed in hot or explosive scenarios leading to the production of new nuclides from previously formed heavier ones by dissociation in the thermal photon bath. This is likely to be the main path for the generation of the approximately 30-40 neutrondeficient nuclides which cannot be produced in neutron capture reactions [1]. For the understanding and modelling of this so-called p-process the dipole strength function up to and near the particle thresholds has to be known accurately [2]. As shown previously [3], details of the dipole strength (now in n-rich nuclei) may as well have large consequences for the r-process path and also s-process branchings are influenced by nuclear excitations [4] induced by thermal photons.

The experimental knowledge [5] on dipole strength is reasonably well established for many heavy and medium mass nuclei in the region of the giant dipole resonance (GDR) by $(\gamma, \mathrm{xn})$ studies, which often also cover the region directly above the neutron threshold $S_{n}$. At lower energies three features have been discussed to be of importance for processes in high-temperature cosmic environments:

a) the fall-off [6-9] of the E1-strength on the low-energy slope of the GDR;

b) the E1-strength between the ground-state (gs) and low energy excitations and its proper extension [10-12] into the regime (a);

c) the occurrence of additional pygmy-resonances, [3,1315], which are assumed to be not as broad as the GDR, but wider as compared to the average level distance $D$ - thus forming an intermediate structure enclosing many levels. Their low energy may well enhance their contribution to photo-dissociation processes in spite of their relatively low strength as compared to the GDR.

In principle, also M1-transitions contribute to the dipole strength, but the average M1 strength is typically 1-2 orders of magnitude smaller as compared to E1, and they are frequently $[1-3]$ not taken into account.

A further approximation has to be introduced to estimate dipole strengths for transitions not connected to the ground state; there are two possibilities proposed in the literature:

a) Strictly following a hypothesis set up by Brink and Axel [16] the strength of a transition connecting in a given nucleus two levels separated by an energy difference $E_{\gamma}$ only depends on $E_{\gamma}$, on the transition type and on a statistical factor determined by the two spins.

b) As proposed by Kadmenskii [10], a temperature dependence of the strength function is introduced, which smears out the GDR-strength into the region below and effectively connects a certain excitation region above the ground state to the GDR domain.

A combination of the two prescriptions has been tried [12], but this suffers from an inconsistency which may be of 
Rusev, Grosse et al.: Pygmy dipole strength in Mo-isotopes

principal nature: At low energy the nuclear excitation is quantized, whereas with increasing energy statistical concepts from thermodynamics are more effective in describing the increasing complexity. Microscopic calculations $[8,11]$ may allow to develop a consistent transition from the low to the high excitation region near the GDR and to properly account for other intermediate strength.

\section{Nuclear resonance fluorescence}

Information about energy dependent dipole strength functions can be obtained from data on multi-step gammadecays following n-capture [17] or direct reactions [12], from inelastic electron scattering [18] or elastic photon scattering $[14,15,19]$. For the region above the n-threshold the electromagnetic strength in a very large number of stable nuclei has been experimentally determined by observing the neutrons emitted after the excitation by quasimonochromatic photons $[5,20]$. Data on this $(\gamma, \mathrm{xn})$ process, taken as averages over a certain energy bin, allow to determine an (averaged) dipole strength function $f_{1}$ in this region by making use of the relation:

$$
f_{1}(E)=\left(3 \pi^{2} \hbar^{2} c^{2} E\right)^{-1} \cdot \sigma_{\gamma}(E)
$$

where $\sigma_{\gamma}$ describes the dipole dissociation of a spin 0 target by a photon of energy $E$. The strength function data obtained from the other methods suitable for the lower excitation energy should connect smoothly to $(\gamma, \mathrm{xn})$ data to yield information about the dipole strength over a wide energy range from the ground state up to far above the particle emission thresholds. Of special importance here is the much discussed $[7,10-12]$ question, if the extrapolation of the Lorentzian fit to the GDR fits dipole strength data also at and below the neutron threshold.

One experimental method has delivered interesting information about nuclear dipole strength; results from it have become more and more detailed with the improving measurements. Using a bremsstrahlung beam the scattering of photons with energies up to the GDR is observed by large volume Ge-detectors. The good resolution $(3-5 \mathrm{keV})$ of such detectors in combination with Compton suppression shields limit the detector response matrix such that it becomes nearly completely diagonal. Thus the signal from elastically scattered photons identifies the energy of the incoming photon out of the bremsstrahlung continuum, as all nuclear levels with sufficient transition strength to the ground-state are observed as narrow elastic scattering resonances up to the respective neutron emission thresholds; inelastic scattering to levels above the target ground states is also observed [21] and has to be considered in the data analysis.

From the knowledge of inelastic scattering via higher lying levels the feeding to a certain level can be accounted for in the analysis of the elastic cross section $\sigma_{\gamma \gamma}$ :

$$
I\left(E_{R}\right)=\int_{R} \sigma_{\gamma \gamma}(E) d E-I_{\text {feed }}
$$

with the integral taken over the (narrow) resonance $\mathrm{R}$ centered at $E_{\gamma}=E_{R}$. The ground state (spin 0) width $\Gamma_{0}$ of such a resonance (spin 1$)$ and $I$ are related by

$$
I\left(E_{R}\right)=3\left(\frac{\pi \hbar c}{E_{R}}\right)^{2} \cdot \frac{\Gamma_{0}^{2}}{\Gamma}
$$

where $\Gamma=\Gamma_{0}+\Gamma_{c}$ is its total width and $\Gamma_{c}$ the summed width of all decay processes competing to the decay back into the ground state. The contribution of the $N_{\Delta}$ resonances in an energy interval $\Delta$ to the dipole strength function can be calculated by:

$$
f_{1, \Delta}=\frac{1}{\Delta} \sum_{i=1}^{N_{\Delta}} \frac{\Gamma_{0, i}}{E_{R, i}^{3}}
$$

The present study on the Mo isotopes uses eq. (4) only for the single line spectra [21] observed below $E_{R} \simeq 4 \mathrm{MeV}$ with low endpoint bremsstrahlung, the other data require a more sophisticated analysis.

A non negligible contribution to the scattering results from non-nuclear processes as Compton scattering with the subsequent bremsstrahlung reaching the detector; pair production leads to photon background in a similar way. Such processes can be simulated [19] to high accuracy and subtracted from the data as shown in Figs. 1 and 2 to yield the "true" nuclear resonance fluorescence (nrf) cross section $\sigma_{\gamma \gamma}$ to be used in eq. (21). Only the resulting difference spectra contain information about the nuclear dipole strength. As the direct contributions from Thomson and Delbrück scattering are weaker by orders of magnitude, the scattering of $\mathrm{MeV}$ photons is primarily nrf. Only when $\Gamma_{c}$ completely dominates $\Gamma$ - e.g. above the neutron threshold $S_{n}$ - the contribution of these direct scattering processes can no longer be neglected. Contributions to the spectra from higher multipole radiation are identified by a differing angular distribution. M1-transitions can be identified through the use of linearly polarized photons $[23-26]$.

\section{Photon scattering experiments on Mo-isotopes}

The present paper reports on photon scattering experiments for the Mo isotopes with $A=92,98$ and 100. This rather wide range in neutron number $N$ may allow a reasonable extrapolation to unstable isotopes; for the case of the pygmy resonance the E1-strength has been predicted [13] to vary strongly with $N$ whereas its energy should weakly depend on $N$. The low- $N$ stable Mo isotopes are p-process nuclides with a surprisingly high cosmic abundance thus making accurate information on the response of Mo-isotopes to dipole radiation especially desirable. The photon scattering experiment at the new radiation source ELBE with its superconducting electron linac [22] was set up similar to previous nrf-studies [13-15,27]. One of its special features is, that the bremsstrahlung emerges from a thin Nb-foil (approx. $5 \mathrm{mg} / \mathrm{cm}^{2}$ ) bombarded with a 


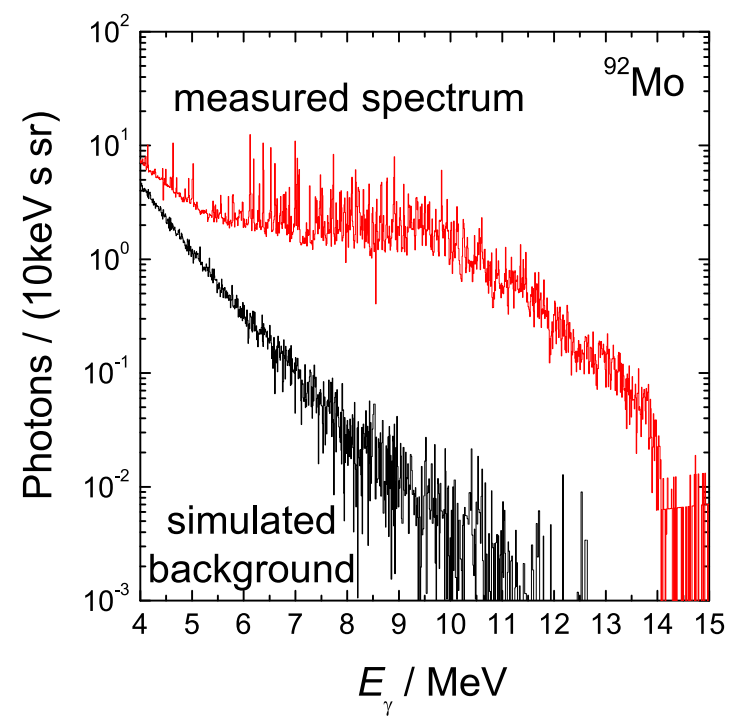

Fig. 1. Spectrum of bremsstrahlung photons scattered by ${ }^{92}$ Mo into $127^{\circ}$. Above a background caused by atomic processes - whose height was determined from a Monte-Carlo simulation - an accumulation of sharp lines near $7 \mathrm{MeV}$ is observed as well as a strong quasi-continuum extending up to the endpoint energy of $\sim 14 \mathrm{MeV}$. Note that lines from identified background sources have been subtracted.

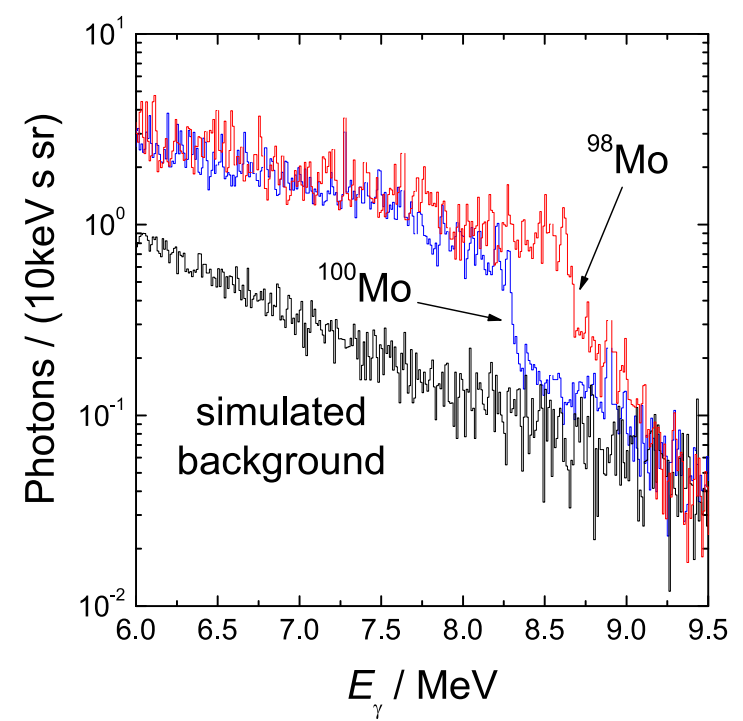

Fig. 2. Same as Fig. 1 for ${ }^{98}$ Mo and ${ }^{100}$ Mo. The background simulation is the same for both isotopes. This allows a test of the determination of the continuum in the region between the two (different) neutron thresholds.

beam of approx. $650 \mu \mathrm{A}=4 \cdot 10^{15} \mathrm{e} / \mathrm{s}$. The electron beam is deflected into a well shielded beam dump after passing the radiator. Highly enriched targets of ${ }^{92} \mathrm{Mo},{ }^{98} \mathrm{Mo}$ and ${ }^{100}$ Mo have been used with masses of 2-3 g each. Electrons with an average momentum of $14 \mathrm{MeV} / \mathrm{c}$ and an rms momentum spread of $0.07 \mathrm{MeV} / \mathrm{c}$ were used in these experiments. For each target a second run with a lower electron momentum was performed such that the endpoint of the bremsstrahlung continuum stayed below the neutron and proton emission thresholds. The photon beam was limited transversely by a $2.5 \mathrm{~m}$ long conical Al-collimator, such that an approximate photon flux of about $10^{7} \gamma /\left(\mathrm{s} \cdot \mathrm{cm}^{2}\right.$. $\mathrm{MeV}$ ) hits the experimental targets. Four large high-purity Germanium semiconductor detectors (enclosed by antiCompton shields from BGO) set up at $90^{\circ}$ and $127^{\circ}$ registered the photons scattered by the target.

The detectors were shielded from unwanted background radiation by lead bricks positioned around the photon beam dump, near the collimator exit and around the beam tube at the target. A conical opening between target and Ge-detector, which was filled by only $2 \mathrm{~cm}$ of $\mathrm{Pb}$ to absorb the very intense low energy photons, determined the angle of observation and the solid angle. Details of the setup and of the Monte-Carlo simulations performed with the aim to optimize it are described elsewhere [19]. According to these simulations the main background contribution to the spectra of scattered photons (as shown in Fig. (1) is due to bremsstrahlung produced from pair production and Compton scattering in the target. The simulation of this background (cf. Fig. 1 and 2) could be made sufficiently accurate to allow the generation of pure nrf-spectra by subtracting the simulated non-resonant contribution from the experimental data.

Due to the $300 \mathrm{keV}$ difference of the neutron binding energies of ${ }^{98} \mathrm{Mo}$ and ${ }^{100} \mathrm{Mo}$ a subtraction of the ${ }^{100}$ Mo data from the ${ }^{98} \mathrm{Mo}$ data results in a pure nrf-spectrum in the range $S_{n}\left({ }^{100} \mathrm{Mo}\right)$ to $S_{n}\left({ }^{98} \mathrm{Mo}\right)$ - under the suggestive assumption that the non resonant background is the same for both isotopes (see Fig. 22). From the fact, that this procedure leads within errors to the same nrf-strength in this energy bin as the Monte-Carlo based subtraction, a test of the accuracy of the latter procedure is obtained. It should be noted here, that the subtraction explained above was performed with the data after their complete correction for detector response. Thanks to the high full energy efficiency of the Ge-detectors used and as result of the good Compton suppression by the BGO shields such a response correction could be performed without introducing large statistical uncertainties.

\section{Dipole strength in isolated narrow resonances}

In the nrf-spectra obtained from the raw data as shown in Figs. 1] and 2 many isolated resonances could be analysed in the range from $4 \mathrm{MeV}$ up to the endpoint energy of 13.2 $\mathrm{MeV}$; the lower part of the spectra is discussed elsewhere [21]. For the three isotopes 299, 310 and 296 resonances, respectively, could be distinguished above $4 \mathrm{MeV}$; no strong lines can be identified above the respective neutron threshold. The ratios of the intensities

$$
\frac{d I}{d \Omega}=\int_{R} \frac{d \sigma(E)}{d \Omega} d E
$$

where the integral includes the full (narrow) resonance $\mathrm{R}$, as observed at scattering angles $90^{\circ}$ and $127^{\circ}$ have been 


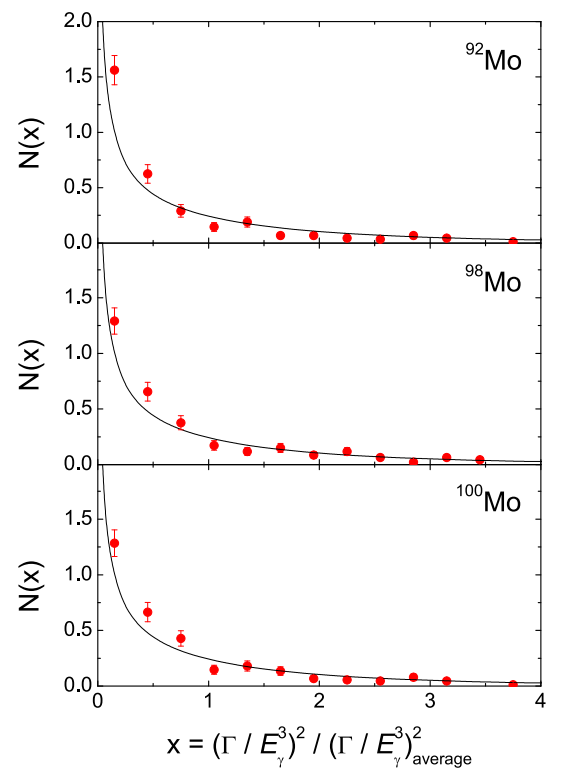

Fig. 3. Distributions of the transition widths as determined from each 50 transitions starting at $4 \mathrm{MeV}$, all reduced by the phase space factor $E_{\gamma}^{3}$. The drawn lines depict Porter-Thomas distributions.

compared to the values expected for a spin sequence 01-0 or 0-2-0 for excitation and deexcitation. Apparently nearly all of the transitions are due to spin 1 resonances. They are assumed to be E1, as from a previous experimental nrf-study on ${ }^{92}$ Mo only 1 resonance is identified [23] to have positive parity; its M1-strength to the gs corresponds to $0.23 \mu_{N}^{2}$ : As in the neighbouring nucleus ${ }^{90} \mathrm{Zr}$ a total M1 strength of $6.7 \mu_{N}^{2}$ was found [24] to lie between 8 and $11 \mathrm{MeV}$, it is likely that a few more M1 resonances are also present in ${ }^{92} \mathrm{Mo}$, but in any case most of the nrfstrength clearly is of E1 character. A similarly low M1 strength has been observed for ${ }^{116} \mathrm{Sn}$ and ${ }^{124} \mathrm{Sn}[26]$ and proposed for $N=82$ nuclei [15].

For the well identified resonances the reduced width distributions are displayed for the three isotopes in Fig. 3 after a normalization to the respective average width taken over bins containing 50 dipole resonances each, observed in the range from $4 \mathrm{MeV}$ up to the neutron binding energies. The distributions are in full agreement to Porter-Thomas distributions indicating chaotic statistics in the ground state transition strengths.

To study as well the statistical properties of the nearest neighbour spacings we also treat the resonances above $4 \mathrm{MeV}$ in groups of 50 and determine the average spacing in each group. The actual spacings divided by this average are shown as black dots in Fig. 4 in comparison to Wigner distributions.

From comparison to the data taken at lower end-point energy it is obvious that above $5 \mathrm{MeV}$ nearly all of the identified transitions connect to the ground state. To obtain an estimate of the possible corrections necessary to account for the incorrect interpretation of non-gs transition energies as level energies we have performed respective Monte Carlo simulations of level sequences describing

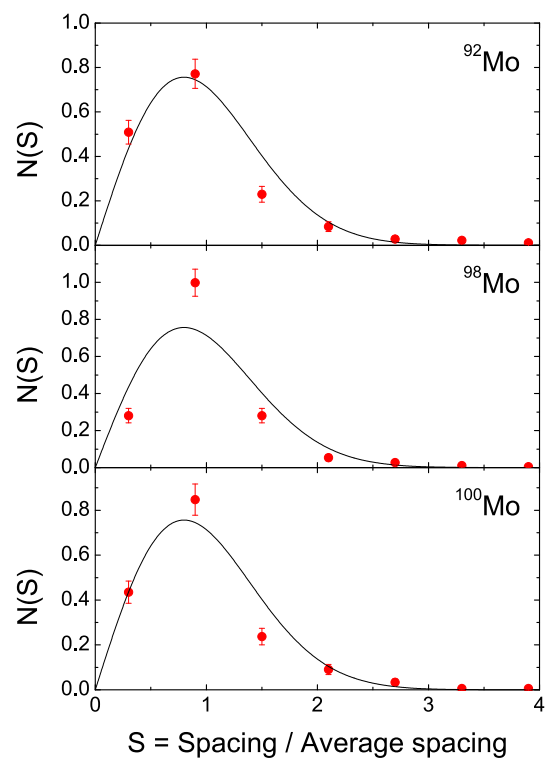

Fig. 4. Next neighbour distance distributions of the isolated narrow resonances above $4 \mathrm{MeV}$. Averages were formed over each 50 transition energies and the ratios of distance to average distance were collected in 7 bins. The drawn lines represent a Wigner distribution.

a Porter-Thomas or a Poisson case. Only a small distortion is caused, when the transition energies resulting from these simulations are (eventually erroneously) treated as level energies. In any case, our Mo data do closely resemble Wigner distributions, again pointing to chaotic statistics.

\section{Level densities and fluctuating cross sections}

The high level density in combination with Porter-Thomas fluctuations cause a large portion of the strength to appear in many weak transitions which are likely to be missed experimentally. This is why an average level density in its dependence on the excitation energy can only be determined from a fluctuation analysis on the basis of PorterThomas statistics [18]. Similarly, the dipole strength in a certain energy interval has to be obtained by integrating the complete nrf spectra - i.e. all counts in discrete resonances and in the quasi-continuum in between, after subtraction of the non-resonant background. The accuracy of the determination of this background can be judged from what is presented in Fig. 2 to be sufficiently high. As obvious from the analysis of the data shown in Figs. [1 and 2 the average strength in the last $\mathrm{MeV}$ below $S_{n}$ is approximately increased by at least a factor 3 when the continuum is included.

Another important correction has to account for inelastic scattering, i.e. transitions branching to excited states. Its effect can be identified from data taken at different endpoint energies of the bremsstrahlung spectrum. When the ${ }^{100} \mathrm{Mo}$ data taken with an endpoint energy of $8.3 \mathrm{MeV}$ 


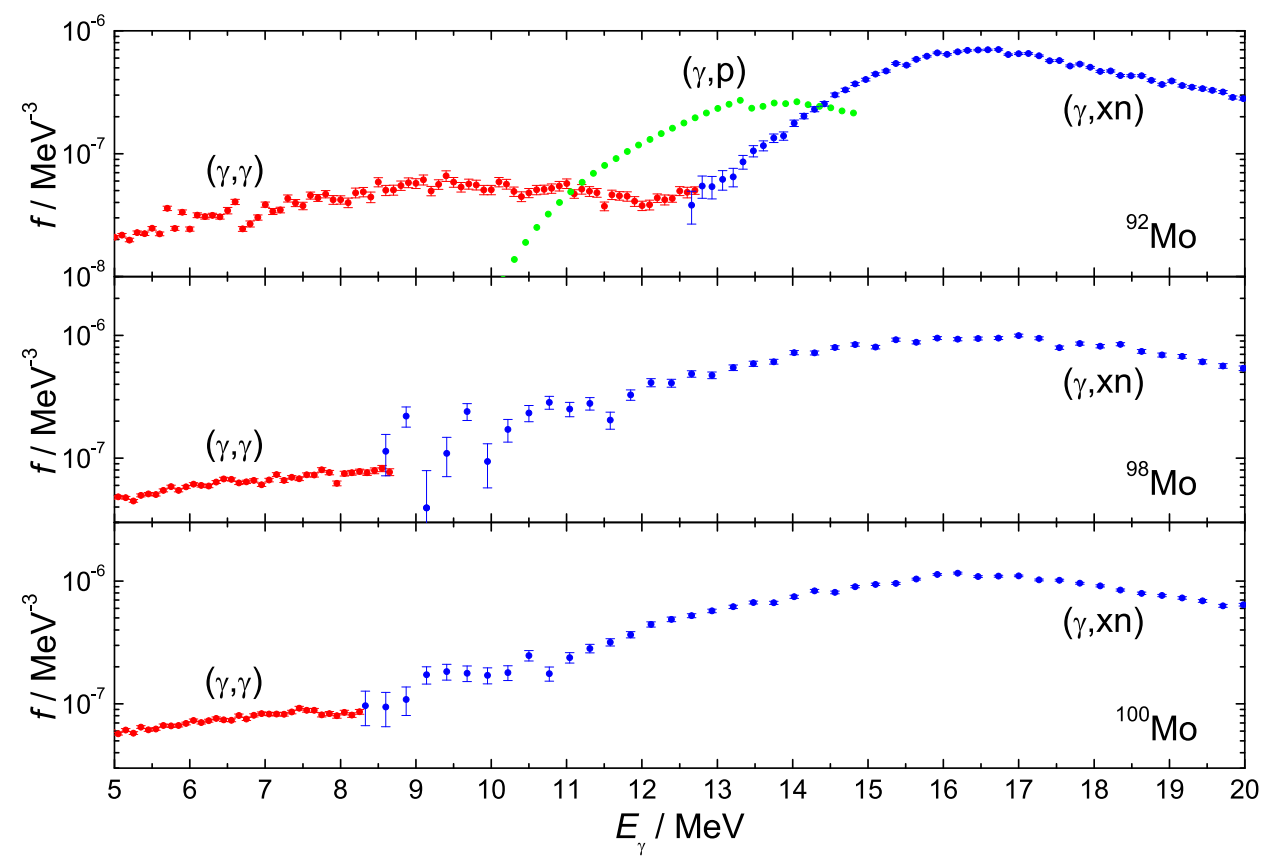

Fig. 5. Dipole strength functions determined from the photon scattering and the $(\gamma, \mathrm{xn})$-data as described in the text. The nrf data are shown in bins of $100 \mathrm{keV}$; this makes the enhancement of strength in several single resonances near $6 \mathrm{MeV}$ less obvious. In the case of ${ }^{92} \mathrm{Mo}$ also the $(\gamma, \mathrm{p})$-process has to be accounted for; this is indicated by including a respective cross section calculation [30] for the corresponding contribution to be added to obtain the total strength. Due to the weakness of quadrupole excitations the plotted $f$ is effectively the dipole strength function $f_{1}$. are compared to the data reaching up to the threshold of $7.8 \mathrm{MeV}$, the intensity distribution originating from these extra $500 \mathrm{keV}$ of bremsstrahlung can be identified: More than $50 \%$ of this intensity is observed as cascades with photons in the range of 3 of $5 \mathrm{MeV}$. Obviously the remaining intensity observed as gs-transitions has to be multiplied by a factor of 2-3 to obtain the full excitation strength. To obtain an approximation for this correction factor the assumption $[27,28]$ is adopted, that below $S_{n}$ and $S_{p}$ inelastic processes (i.e. branching) can on the average be accounted for by setting $\Gamma_{c}=0.2 \mathrm{eV}$ (for the $\mathrm{Zr}$ region) in combination with the level density [29]. A more accurate correction is to be gained from the experiments at lower energy and by Monte-Carlo-simulations; obviously the approximation applied as described above cannot introduce extra structures.

This is the first time, that high resolution nrf-spectra are analyzed such that not only the isolated resonances are included, but also the fluctuating quasi-continuum. This is accomplished by calculating the dipole strength function for the energy range up to $S_{n}$ (as covered in this experiment) from the elastic component $\sigma_{\gamma \gamma}$ of nrf:

$$
f_{1}(E)=\left(3 \pi^{2} \hbar^{2} c^{2} E\right)^{-1} \cdot \frac{\Gamma}{\Gamma_{0}} \cdot \frac{1}{\Delta} \cdot \int_{\Delta} \sigma_{\gamma \gamma}\left(E_{\gamma}\right) \cdot d E_{\gamma}
$$

where $\Delta$ is the interval selected around $E$ for averaging the widths $\Gamma_{0}$ and $\Gamma$ and the photon energy $E_{\gamma}$. The photon absorption cross section is thus derived from the observed elastic photon scattering cross section $\sigma_{\gamma \gamma}$ by correcting bin-wise for inelastic scattering (i.e. branching). This determination of $f_{1}$ then allows a quantitative comparison of nrf - and ( $\gamma, \mathrm{xn})$ - data (cf eq. 1), and both can be directly combined to extract a continuous dipole strength function.

\section{The distribution of photon strength and pygmy resonances}

The good compatibility of the nrf-strength (corrected for branching) directly below $S_{n}$ and the $(\gamma, \mathrm{xn})$-data directly above encourages a search for structure in $f_{1}(E)$ derived from the two data sets. The data indicate an enhancement of the dipole strength at $\sim 9 \mathrm{MeV}$. In ${ }^{92} \mathrm{Mo}$ this possible pygmy resonance appears below and in ${ }^{100} \mathrm{Mo}$ it is above $S_{n}$; in ${ }^{98} \mathrm{Mo}$ the region directly above $S_{n}=8.6 \mathrm{MeV}$ shows some irregularities. In an old tagged photon scattering experiment on natural $\mathrm{Zr}$ cross section enhancements at 9.1 and $11.6 \mathrm{MeV}$ were found [27]; from the isotope enrichment and the n-threshold values it is argued, that they should originate from ${ }^{90} \mathrm{Zr}$. Most of that strength was shown not to be M1 $[24,28]$. The Mo-data from ELBE as well as these results have to be compared to broad resonance-like structures seen [26] in Sn isotopes at 6.7 and between 8.0 and $8.7 \mathrm{MeV}$. The strength as extracted from an experiment [26] on ${ }^{116} \mathrm{Sn}$ an ${ }^{124} \mathrm{Sn}$ clearly stays below the extrapolation of the GDR-Lorentzian as only narrow isolated resonances had been analyzed. Although the broad structures seen in Sn by the tagged photon study [27] and in the new Ge-detector experiment [26] appear at nearly the same energies, the strength observed in the region of the broad pygmy structure differs by a factor of two between the two types of experiment. Obviously, an analysis of the well isolated peak on the basis of eq. (4) misses much of the strength which is accounted for by following a procedure characterized by eq. (6), which does not ignore the fluctuating quasi-background. The only other isotope chain studied in this range of $A$, the even Geisotopes, show [25] no clear pygmy resonance appearing 
in the discrete spectra below $S_{n}$. The average strength function obtained from these resonances by using formula (41) amounts to $\sim 10^{-8} \mathrm{MeV}^{-3}$ and clearly stays below the extrapolated Lorentzian extracted from the $(\gamma, \mathrm{xn})$-data. Recent HFB-QRPA calculations [8] give good fits to GDR data when the force Bsk-7 of Skyrme-type is used to describe the effective nucleon-nucleon interaction. Nevertheless it should be noted here, that $\sigma(\gamma, \mathrm{n})$ directly above threshold is well described only for ${ }^{94} \mathrm{Mo}$, whereas the calculation is below the experimental value [20] for ${ }^{100} \mathrm{Mo}$ by a factor 4 . An extension of such calculations to lower $E$ including the pygmy region - seems interesting.

\section{Conclusion}

The response of nuclei to dipole radiation can well be studied by photon scattering investigated at a bremsstrahlung facility like ELBE. Using a sufficiently high endpoint energy and correcting the data for inelastic processes allows to directly combine the dipole strength functions $f_{1}$ obtained from the $(\gamma, \gamma)$ (i.e. nrf) and the $(\gamma, \mathrm{xn})$ data; together they span the full range from the ground state to the GDR. A comparison of $f_{1}(E)$ to a Lorentzian extrapolated from the GDR needs a more thorough discussion of the spreading of the GDR than is accomplished by just fitting near its maximum $[5,20]$. Apparently the large apparent width of the GDR in ${ }^{100}$ Mo may be caused by a deformation of that nucleus; accounting for that by a two-resonance-fit would reduce the low energy tail considerably. Calculations of the type presented recently $[2,8,11]$ may help to clarify this point, especially when the nuclear deformation is included with sufficient accuracy.

Above $\sim 4 \mathrm{MeV}$ the predicted level densities [29] increasingly surpass the number of identifiable resonances and apparently the levels of ${ }^{92} \mathrm{Mo},{ }^{98} \mathrm{Mo}$ and ${ }^{100} \mathrm{Mo}$ show signs of a chaotic structure: The next neighbour distance distributions of the clearly identified peaks are Wigner distributed and their ground-state transition widths follow Porter-Thomas distributions. From these facts one expects the photon scattering excitation functions - which in a bremsstrahlung beam are observed simultaneously over a wide range - to show Porter-Thomas fluctuations in case the detector resolution surpasses the average peak distance. As this is the case for most of the Mo-data discussed here, a reasonable extraction of strength information should not ignore the fluctuating quasi continuous part of the cross section.

Thus $f_{1}(E)$ was determined for the three Mo-isotopes by using all scattering strength with the exception of the nonresonantly scattered photons, whose contribution to the spectra was calculated and subtracted. The $f_{1}$-data for the three Mo isotopes show a clear maximum at $\sim 9 \mathrm{MeV}$ indicating the presence of a pygmy resonance, as it was observed at this energy also in ${ }^{90} \mathrm{Zr}$ and slightly lower in energy in $\mathrm{Sn}$ isotopes. The $9 \mathrm{MeV}$-structure is below $S_{n}$ in ${ }^{92} \mathrm{Mo}$ and clearly above $S_{n}$ in ${ }^{100} \mathrm{Mo}$; in ${ }^{98} \mathrm{Mo}$ a cross section irregularity shows up at the neutron threshold. Intermediate structures are weakly showing up also between 6 and $7 \mathrm{MeV}$ (as in ${ }^{118} \mathrm{Sn}$ and ${ }^{124} \mathrm{Sn}$ ) and eventually also at
$11 \mathrm{MeV}$ (as in ${ }^{90} \mathrm{Zr}$ ). This may be considered an indication for a sequence of pygmy resonances - not just one.

\section{Acknowledgements}

Dr. P. Michel and the ELBE-Crew made these experiments possible with their strong commitment to deliver optimum beams. A. Hartmann and W. Schulze provided very valuable support during the difficult experiments. Intensive discussions with Dr.F. Becvar, Dr.F. Dönau and Dr. R. Wünsch are gratefully acknowledged. The DFG has supported one of us (G. R.) under Do466/1-2 during the course of the studies presented here.

\section{References}

1. E.M. Burbridge et al.: Rev. Mod. Phys. 29, (1957) 547; M. Arnould and S. Goriely, Phys. Rep. 384, (2003) 1;

T. Hayakawa et al.: Phys. Rev. Lett. 93, (2004)

2. S. Goriely and Khan: Nucl. Phys. A706, (2002) 217

3. S. Goriely: Phys. Lett. B436, (1998) 10

4. F. Käppeler et al.: Rep. Progr. Phys. 52, (1989) 945

5. S.S. Dietrich and B.L. Berman: At. Data Nucl. Data Tables 52, (1989) 199;

B.L. Bermann et al.: Phys. Rev. C36, (1987) 1286

6. C.M. McCullagh et al.: Phys. Rev. C23, (1981) 1394

7. I. Kopecky and R.E. Chrien: Nucl. Phys. A468, (1987) 285; I. Kopecky and M. Uhl: Phys. Rev. C41, (1990) 1941

8. S. Goriely et al.: Nucl. Phys. A739, (2004) 331

9. H. Utsonomiya et al.: Phys. Rev. C67, (2003) 015807

10. S.G. Kadmenskii et al.: Sov. J. Nucl. Phys. 37, (1983) 165

11. M. Arnould and S. Goriely, accepted by Nucl. Phys. A doi:10.1016/j.nuclphysa.2005.02.116

12. M. Guttormsen et al.: Phys. Rev. C71, (2005) 044307 and refs. therein

13. P. van Isacker et al.: Phys. Rev. C45, (1992) R13

14. N. Ryezayeva et al.: Phys. Rev. Lett. 89, (2002) 272501

15. R.D. Herzberg et al.: Phys. Rev. C60, (1999) 051307; A. Zilges et al.: Progr. in Part. and Nucl. Phys. 55, (2005) 408

16. D.M. Brink: Ph.D.thesis, Oxford University, (1955); P. Axel: Phys. Rev. 126, (1962) 671

17. L. Zanini et al.: Phys. Rev. C68, (2003) 014320; M. Krticka et al.: Phys. Rev. Lett. 92, (2004) 172501

18. A. Richter: Phys. Scr. T5, (1983) 63; G. Kilgus et al.: Z. Phys. A326, (1987) 326; P.G. Hansen et al.: Nucl. Phys. A518, (1990) 13

19. R. Schwengner et al.: submitted to Nucl. Instr. and Meth.

20. H. Beil et al.: Nucl. Phys. A227, (1974) 427, corrected according to ref. 5 .

21. G. Rusev et al.: Phys. Rev. Lett., in print; G. Rusev et al.: to be published

22. J. Teichert et al.; Nucl. Instr. and Meth. A507, (2003) 354

23. F. Bauwens, Dissertation Univ. Gent (B) (2000)

24. R.M. Laszewski et al.: Phys. Rev. Lett. 59, (1987) 431

25. A. Jung et al.: Nucl. Phys. A584, (1995) 103

26. K. Govaert et al.: Phys. Rev. C 57, (1998) 2229

27. P. Axel et al.: Phys. Rev. C2, (1970) 689

28. R. Alarcon et al.: Phys. Rev. C36, (1987) 954

29. D. Bucurescu and T. von Egidy: J. of Phys., in press

30. T. Rauscher and F.-K. Thielemann: At. Data Nucl. Data Tables 88, (2004) 1 